\title{
Self-accelerated Universe
}

\author{
B. P. Kosyakov \\ Russian Federal Nuclear Center-VNIIEF, Sarov 607190, Russia
}

\begin{abstract}
It is widely believed that the large redshifts for distant supernovae are explained by the vacuum energy dominance, or, in other words, by the cosmological constant in Einstein's equations, which is responsible for the anti-gravitation effect. A tacit assumption is that particles move along a geodesic for the background metric. This is in the same spirit as the consensus regarding the uniform Galilean motion of a free electron. However, there is a runaway solution to the Lorentz-Dirac equation governing the behavior of a radiating electron, in addition to the Galilean solution. Likewise, a runaway solution to the entire system of equations, both gravitation and matter equations of motion including, may provide an alternative explanation for the accelerated expansion of the Universe, without recourse to the hypothetic cosmological constant.
\end{abstract}

\section{Introduction}

The recent measurements of redshifts for Type Ia supernovae [1] suggest that the Universe expansion is accelerating. To interpret this discovery, one usually write the Friedmann equation

$$
H^{2}=\left(\frac{\dot{a}}{a}\right)^{2}=\frac{2}{A_{V}^{2}}+\frac{2 A_{D}}{a^{3}}+\frac{2 A_{B}}{a^{3}}+\frac{A_{R}}{a^{4}}-k
$$

where $H$ is the Hubble expansion parameter, $a$ is the scale factor, $A_{V}, A_{D}, A_{B}$, and $A_{R}$ are Friedmann integrals of the motion related to the energy density of vacuum, dark matter, nonrelativistic particles (barions), and radiation, respectively, $k$ is the spatial curvature, with $k=1,0,-1$ corresponding to the closed, flat, and open models. Equation (1) is derived from Einstein's equations with a positive cosmological constant

$$
R_{\mu \nu}-\frac{1}{2} g_{\mu \nu} R-\Lambda g_{\mu \nu}=-8 \pi G T_{\mu \nu}
$$

using the generic form of the line element for homogeneous and isotropic spacetimes

$$
d s^{2}=d t^{2}-a^{2} F(r)^{2} d \Omega^{2}-a^{2} d r^{2} .
$$

Here, $t$ is the proper time, $d \Omega=d \theta^{2}+\sin ^{2} \theta d \varphi, F=\sin r, r, \sinh r$ for $k=1,0,-1$, respectively, the cosmological constant $\Lambda$ relates to the vacuum energy density $\rho_{V}$ as $\Lambda=8 \pi G \rho_{V}$, and $A_{V}=\left(8 \pi G \rho_{V} / 3\right)^{-1 / 2}$. In the expanding Universe, the scale factor $a$ increases with time. So, there comes a time when the first term in (1) becomes dominant. The asymptotic $a \rightarrow \infty$ solution to Eq. (1) is

$$
a(t)=A_{V} f(t), \quad f(t)=\cosh \left(t / A_{V}\right), \exp \left(t / A_{V}\right), \sinh \left(t / A_{V}\right) \quad \text { for } \quad k=1,0,-1 .
$$

This solution describes a cosmological expansion accelerating in time, $\ddot{a}>0$.

Thus the presence of a positive cosmological constant $\Lambda$ in (2), which is responsible for the antigravitation effect, ensures the accelerating expansion regime of the Universe. At present, this explanation of the large redshift data for distant supernovae is widely accepted. It tacitly assumes that particles (galaxies, clusters, etc.) move along geodesic for the background metric. Recall, however, that galaxies every so often have an internal angular momentum (spin), and a spinning particle is deflected from the geodesic. It is clear that the spacetime curvature is of no concern: a particle with spin can behave in a non-Galilean manner in a flat spacetime. Let us consider the Frenkel model of a classical spinning particle [2]. Its motion, in the absence of external forces, is governed (see, e. g., [3]) by the equation

$$
\mathcal{S}^{2} \ddot{v}^{\mu}+M^{2} v^{\mu}=m p^{\mu}
$$


where $\mathcal{S}$ is the spin magnitude, $v^{\mu}=\dot{z}^{\mu}$ is the four-velocity, the dot denotes the derivative with respect to the proper time $s, p^{\mu}$ is the four-momentum (which is constant for the free particle), $M$ and $m$ are the mass and rest mass defined as $M^{2}=p^{2}$ and $m=p \cdot v$. For $p^{2}>0, p^{\mu}=$ const, a solution to Eq. (5) is

$$
z^{\mu}(s)=\frac{m}{M^{2}} p^{\mu} s+\frac{\alpha^{\mu}}{\omega} \cos \omega s+\frac{\beta^{\mu}}{\omega} \sin \omega s
$$

where $\alpha \cdot p=\beta \cdot p=\alpha \cdot \beta=0, \alpha^{2}=\beta^{2}$. This helical world line describes the motion called the Zitterbewegung [4]. For $p^{2}<0, p^{\mu}=$ const, we have

$$
z^{\mu}(s)=-\frac{m}{\mathcal{M}^{2}} p^{\mu} s+\frac{\alpha^{\mu}}{\Omega} \cosh \Omega s+\frac{\beta^{\mu}}{\Omega} \sinh \Omega s
$$

where $\mathcal{M}^{2}=-p^{2}, \Omega=\mathcal{M} / \mathcal{S}$, and $\alpha^{\mu}$ and $\beta^{\mu}$ are subjected to the constraint $\alpha^{2}=-\beta^{2}$. This solution describes the motion with increasing velocity.

One may argue that spacelike four-momenta $p^{\mu}$ are highly unnatural for classical particles. While this is a strong objection, it seems reasonable to say that both solutions (6) and (7) support the idea of non-Galilean regimes for free spinning particles.

Another fact deserving of notice is that massive particles can emit gravitational waves. It is conceivable that a massive particle emitting gravitational waves moves in a runaway regime, that is, deviates sharply from a geodesic for the background metric $g_{\mu \nu}$. Runaway solutions offer an alternative explanation for the accelerated expansion of the Universe, without recourse to the cosmological constant hypothesis.

It is interesting to compare a massive particle emitting gravitational waves and a charged particle emitting electromagnetic waves. The nonrelativistic equation of motion for a classical electron, called the Abraham-Lorentz equation (see, e. g., [5]),

$$
m \mathbf{a}-\frac{2}{3} e^{2} \frac{d \mathbf{a}}{d t}=\mathbf{f}
$$

in the absence of external forces $\mathbf{f}=0$, becomes

$$
\mathbf{a}-\tau_{0} \frac{d \mathbf{a}}{d t}=0
$$

where

$$
\tau_{0}=\frac{2 e^{2}}{3 m} \approx 10^{-23} \mathrm{~s} .
$$

The general solution to Eq. (9),

$$
\mathbf{a}(t)=\mathbf{A} \exp \left(t / \tau_{0}\right),
$$

where $\mathbf{A}$ is the initial acceleration at $t=0$, describes a runaway motion. For $\mathbf{A}=0$, we have $\mathbf{a}=0$, and $\mathbf{v}=$ const. Thus a free electron can behave as both Galilean $(\mathbf{A}=0)$, and non-Galilean $(\mathbf{A} \neq 0)$ objects.

Where does the Abraham-Lorentz equation come from? The scheme of its derivation is as follows. We first solve Maxwell's equations

$$
\square A^{\mu}(x)=4 \pi e \int_{-\infty}^{\infty} d s v^{\mu}(s) \delta^{4}(x-z(s))
$$

where the world line of a single charge $z^{\mu}(s)$ is taken to be arbitrary timelike smooth curve. The retarded Lienárd-Wiechert solution

$$
A^{\mu}(x)=\left.\frac{e v^{\mu}}{(x-z) \cdot v}\right|_{s=s_{\text {ret }}}
$$

is regularized and substituted to the equation of motion for a bare charged particle

$$
m_{0} \mathbf{a}=e(\mathbf{E}+\mathbf{v} \times \mathbf{B}),
$$

where $m_{0}$ is the bare mass. We then require that the renormalized mass

$$
m=\lim _{\epsilon \rightarrow 0}\left(m_{0}(\epsilon)+\frac{e^{2}}{2 \epsilon}\right)
$$

be a finite positive quantity. Finally, we arrive at the Abraham-Lorentz equation (8) in the limit of the regularization removal $\epsilon \rightarrow 0$. 
In order to derive the equation of motion for a particle, which is capable of emitting gravitational waves, one should repeat the essentials of this procedure: to find a retarded solution to Einstein's equations (2) with $\Lambda=0$ assuming that a given point particle which generates the retarded gravitational field moves along an arbitrary timelike smooth world line, regularize this solution, substitute it to the equation of motion for the bare particle, perform the mass renormalization, and remove the regularization. This will yield the desired equation of motion for the dressed particle, which is apparently different from the equation of a geodesic of the metric $g_{\mu \nu}$. However, this project is highly nontrivial. Even the first stage has still defied implementation: we have no solution to the gravitation equations (2) similar to the Lienárd-Wiechert solution (13) in electrodynamics. So, at the moment we cannot offer a complete explanation for the accelerated expansion of the Universe based on self-accelerated solutions similar to the runaway solution (11) to the Abraham-Lorentz equation. Nevertheless, with results drawn from solvable theories where particles interact with scalar, tensor, Yang-Mills, and linearized gravitational fields, and dimensional considerations, we can construct with some degree of certainty the form of the equation of motion for a particle emiting gravitation waves.

\section{Dressed particles}

The relativistic generalization of the Abraham-Lorentz equation (8) is the Lorentz-Dirac equation (see, e. g., $[6])$

$$
m a^{\lambda}-\frac{2}{3} e^{2}\left(\dot{a}^{\lambda}+v^{\lambda} a^{2}\right)=f^{\lambda} .
$$

It accounts for the dynamics of a synthesized object whose inertia is characterized by the quantity $m$, defined in (15) where mechanical and electromagnetic field terms contribute. We will call this object the dressed charged particle. The state of the dressed particle is specified by the four-coordinate of the singular field point $z^{\mu}$ and the four-momentum

$$
p^{\mu}=m v^{\mu}-\frac{2}{3} e^{2} a^{\mu}
$$

assigned to this point [7]. The singular point is governed by the equation

$$
\stackrel{v}{\perp}(\dot{p}-f)=0
$$

where $\stackrel{v}{\perp}$ is the projection operator

$$
\stackrel{v}{\perp}_{\mu \nu}=\eta_{\mu \nu}-\frac{v_{\mu} v_{\nu}}{v^{2}},
$$

and $f^{\mu}$ is an external four-force applied to the point $z^{\mu}$. Indeed, substitution of (17) in (18) gives the Lorentz-Dirac equation (16). On the other hand, Eq. (18) is nothing but Newton's second law in a coordinate-free form.

Teitelboim [7] was able to show that the Lorentz-Dirac equation (16) is equivalent to the local energymomentum balance,

$$
\dot{p}^{\mu}+\dot{\mathcal{P}}^{\mu}+\dot{\wp}^{\mu}=0,
$$

where the four-momentum of the dressed particle $p^{\mu}$ is defined in (17), the radiation four-momentum $\mathcal{P}^{\mu}$ is represented by the Larmor formula,

$$
\mathcal{P}^{\mu}=-\frac{2}{3} e^{2} \int_{-\infty}^{s} d \tau v^{\mu} a^{2},
$$

and the four-momentum $\wp^{\mu}$ relates to the integral of the external Lorentz four-force,

$$
\wp^{\mu}=-\int_{-\infty}^{s} d \tau f^{\mu} .
$$

The balance equation (20) reads: the four-momentum $d_{\wp}{ }^{\mu}=-f^{\mu} d s$ which has been extracted from an external field during the period of time $d s$ is distributed between the four-momentum of the dressed particle, $d p^{\mu}$, and the four-momentum carried away by the radiation $d \mathcal{P}^{\mu}$.

Let $f^{\mu}$ be zero, Eq. (16) is satisfied by

$$
v^{\mu}(s)=\alpha^{\mu} \cosh \left(w_{0} \tau_{0} e^{s / \tau_{0}}\right)+\beta^{\mu} \sinh \left(w_{0} \tau_{0} e^{s / \tau_{0}}\right)
$$


where $\alpha^{\mu}$ and $\beta^{\mu}$ are constant four-vectors that meet the conditions $\alpha \cdot \beta=0, \alpha^{2}=-\beta^{2}=1, w_{0}$ is an initial acceleration magnitude, and $\tau_{0}$ is given by (10). The solution (23) describes a runaway motion, which becomes a uniform Galilean motion for $w_{0}=0$. We see from (11) and (23) that the class of Galilean world lines is distinct from the class of runaway world lines. A dressed particle may either show itself as a Galilean object or execute perpetually a self-accelerated motion, none of the Galilean objects is able to become self-accelerated and vice versa. The non-Galilean behavior is an innate feature of some species of dressed particles.

It is often asserted that the solution (23) is "unphysical". The fact that because a free particle continually accelerates and continually radiates seems contrary to energy conservation. We note that a mechanical object with the four-momentum $p^{\mu}=m v^{\mu}$ is understood in such claims. By contrast, proceeding from the concept of a dressed particle with the four-momentum defined in (17), we have the balance equation (20). There is no contradiction with energy conservation in this framework: the energy variation of the dressed particle $d p^{0}$ is equal to the energy carried away by the radiation $-\dot{\mathcal{P}}^{0} d s$. A subtlety is that the dressed particle energy

$$
p^{0}=m \gamma\left(1-\tau_{0} \gamma^{3} \mathbf{a} \cdot \mathbf{v}\right)
$$

is not positive definite. The indefiniteness of expression (24) means that increase of the velocity magnitude $|\mathbf{v}|$ need not be accompanied by increase of $p^{0}$.

For $m=0$, which is another reasonable option of the mass renormalization (15), the first term of Eq. (16) disappears. Take $f^{\mu}=0$, then (16) becomes

$$
(\stackrel{v}{\perp} \dot{a})^{\mu}=0,
$$

which is the equation that describes a relativistic uniformly accelerated motion [6]. The world line of a free dressed particle with $m=0$ is a hyperbola

$$
v^{\mu}(s)=\alpha^{\mu} \cosh w_{0} s+\beta^{\mu} \sinh w_{0} s, \quad \alpha \cdot \beta=0, \quad \alpha^{2}=-\beta^{2}=1 .
$$

The curvature $k=w_{0}=$ const of such a world line may be arbitrary. Thus the non-Galilean regime for massless dressed particle is a hyperbolic motion.

It follows from (17) that

$$
M^{2}=p^{2}=m^{2}\left(1+\tau_{0}^{2} a^{2}\right) .
$$

For $\tau_{0}^{2} a^{2}<-1$, the dressed particle turns to a tachyonic state, that is, the state with $p^{2}<0$, during the period of time

$$
\Delta s=-\frac{\tau_{0}}{2} \log \left|\tau_{0}^{2} a^{2}(0)\right| .
$$

This observation gives insight into why the runaway motion of dressed charged particles was never observed. The period of time over which a self-accelerated electron possesses a timelike four-momentum is tiny. From (10) we find $\Delta s \sim \tau_{0} \sim 10^{-23}$ s for electrons, and still shorter for other charged elementary particles. All primordial self-accelerated particles with such $\tau_{0}$ have long been in tachyonic states. However, we have not slightest notion of how tachyons can be experimentally recorded. It seems plausible that primordial self-accelerated particles, which transmuted into tachyons, represent part of dark matter.

If a cosmological object is considered as a dressed particle emitting gravitational waves, the characteristic period $\tau_{0}$ may be found to be comparable with the inverse current Hubble scale $H^{-1}$. The self-acceleration of such an object can indeed be observed at the present time. For clarity, the experimental value of this scale is $H^{-1}=(46 \pm 4) \cdot 10^{16} \mathrm{~s}$.

We now turn to a dressed colored particle in the cold QCD phase [8]. The equation of motion for a dressed quark with the color charge $Q$ in an external $\mathrm{SU}(\mathcal{N})$ Yang-Mills field $F^{\mu \nu}$ is

$$
m\left[a^{\mu}+\ell\left(\dot{a}^{\mu}+v^{\mu} a^{2}\right)\right]=\operatorname{tr}\left(Q F^{\mu \nu}\right) v_{\nu}
$$

where $m$ is the renormalized mass, and

$$
\ell=\frac{8}{3 m g^{2}}\left(1-\frac{1}{\mathcal{N}}\right),
$$

$g$ is the Yang-Mills coupling constant. For $F^{\mu \nu}=0$, the general solution to Eq. (27)

$$
v^{\mu}(s)=\alpha^{\mu} \cosh \left(w_{0} \ell e^{-s / \ell}\right)+\beta^{\mu} \sinh \left(w_{0} \ell e^{-s / \ell}\right)
$$


describes a self-decelerated motion. By contrast, in the hot QCD phase, dressed quarks may execute a runaway motion like (23) or (25).

At first sight, the self-deceleration is an innocent phenomenon, because the motion becomes almost indistinguishable from Galilean in the short run. However, the presence of self-decelerations actually jeopardizes the consistency of the theory: the acceleration increases exponentially as $s \rightarrow-\infty$, and the intensity of the energy gain grows along with it in this limit. The Yang-Mills field energy at any finite instant is therefore divergent.

Let us consider a dressed particle interacting with a massless scalar field [9]. The equation of motion for a dressed particle in an external scalar field $\phi$ is

$$
\frac{d}{d s}(m+g \phi) v^{\mu}-\frac{1}{3} g^{2}\left(\dot{a}^{\mu}+a^{2} v^{\mu}\right)=g \partial^{\mu} \phi
$$

where $m$ is the renormalized mass, and $g$ is the coupling constant.

Likewise, the equation of motion for a dressed particle interacting with an external tensor field $\phi_{\alpha \beta}$ in the linearized gravity $[10,9]$ is

$$
\begin{gathered}
\frac{d}{d s}\left[v^{\mu}\left(1-\frac{1}{2} v^{\alpha} v^{\beta} \phi_{\alpha \beta}-\frac{1}{2} \phi_{\alpha}^{\alpha}\right)+v_{\alpha} \phi^{\alpha \mu}\right]+\frac{11}{3} G m\left(\dot{a}^{\mu}+a^{2} v^{\mu}\right) \\
=\frac{1}{2} v^{\alpha} v^{\beta} \partial^{\mu} \phi_{\alpha \beta}-\frac{1}{4} \partial^{\mu} \phi_{\alpha}^{\alpha}
\end{gathered}
$$

where $G$ is the gravitational constant, and $m$ is the renormalized mass.

If we define the Abraham factor

$$
\Gamma^{\mu}=\dot{a}^{\mu}+v^{\mu} a^{2},
$$

the results regarding the equations of motion for dressed particles can be summarized in the following table

Table 1. Abraham term in different theories

\begin{tabular}{|c||c||c||c|c||c|}
\hline Scalar field & Vector field & $\begin{array}{c}\text { Yang-Mills } \\
\text { (cold phase) }\end{array}$ & $\begin{array}{c}\text { Tensor field } \\
\phi_{\mu \nu}=\lambda \frac{v_{\mu} v_{\nu}}{\rho}\end{array}$ & $\begin{array}{c}\text { Tensor field } \\
\phi_{\mu \nu}=\lambda \frac{\eta_{\mu \nu}}{\rho}\end{array}$ & $\begin{array}{c}\text { Linearized } \\
\text { gravity }\end{array}$ \\
\hline \hline$\frac{1}{3} g^{2} \Gamma^{\mu}$ & $\frac{2}{3} e^{2} \Gamma^{\mu}$ & $-\frac{2}{3}\left|Q^{2}\right| \Gamma^{\mu}$ & $-\frac{5}{3} \lambda^{2} \Gamma^{\mu}$ & $-\frac{1}{3} \lambda^{2} \Gamma^{\mu}$ & $-\frac{11}{3} G m^{2} \Gamma^{\mu}$ \\
\hline
\end{tabular}

\section{Discussion}

For dimensional reasons, we may expect that the equation of motion for a dressed particle emitting gravitational waves should include a covariant generalization of the Abraham term. Meanwhile the sign of the Abraham term in the linearized gravity is negative which implies that the non-Galilean regime of massive particle emitting gravitational waves is self-decelerating, not self-accelerating. Moreover, one can estimate the characteristic time $\tau_{0}$ in (31) taking $m$ to be a typical cluster mass, $\tau_{0}=G m \sim 10^{8} \mathrm{~s} \sim 3$ light years. It is very far from $\tau_{0} \sim H^{-1} \sim 2 \cdot 10^{10}$ light years. These results may appear discouraging in respect to the alternative explanation of the accelerated motion of distant cosmological object. Recall, however that the nonlinearity peculiar to Einstein's equation may change the situation drastically, as the Yang-Mills theory suggests [8]. Two phases are frequent among nonlinear systems: hadron and quarkgluon phases in QCD, laminar and turbulent phases in hydrodynamics are examples. It is conceivable that gravity may also reveal two phases which are classified according to whether the emission of gravitational waves is attended with energy gains or energy losses. In the former phase, the motion of dressed particles is self-decelerated, and in the latter phase, it is self-accelerated. Contributions from interactions with other classical fields (electromagnetic, dilaton, gluon) may also have a dramatic effect on the sign and the magnitude of the aggregate Abraham term. In addition, when on the subject of galaxies and clusters, we should take into account their spins. The description of a radiating charged particle with spin is a challenging problem; it was discarded here to make the key idea as simple as possible (for a review see [11]).

Finally, a remarkable fact is that the characteristic time $\tau_{0}=G m$ with $m$ being the total visible mass of the Universe is of order of the inverse current Hubble scale $H^{-1}$. Does this mean that the Universe as a whole executes a self-accelerated motion?

I would like to thank I. D. Novikov for helpful comments. This work was supported in part by ISTC under the Project \# 840. 


\section{References}

[1] A. G. Riess et al., "Observational evidence from supernovae for an accelerating universe and a cosmological constant," Astron. J. 116, 1009, 1998; astro-ph/9805201; S. Perlmutter et al., "Measurement of Omega and Lambda from 42 high-redshift supernovae," Astrophys. J. 517, 565, 1999; astro-ph/9812133.

[2] J. Frenkel, "Die Elektrodynamik des Rotierenden Electrons," Z. Phys. 37, 243, 1926.

[3] B. P. Kosyakov, "On inert properties of particles in classical theory," hep-th/0208035.

[4] E. Schrödinger, "Über die kräftfreie Bewegung in der relativistischen Quantenmechanik," Sitzungber. Preuss. Acad. Wiss. 24, 418, 1930.

[5] J. D. Jackson, Classical Electrodynamics (New York: Wiley, 1962, 1975, and 1998).

[6] F. Rohrlich, Classical Charged Particles (Reading: Addison-Wesley, 1965, and 1990).

[7] C. Teitelboim, "Splitting of Maxwell tensor: Radiation reaction without advanced fields," Phys. Rev. D 1, 1572, 1970.

[8] B. P. Kosyakov, "Exact solutions in the Yang-Mills-Wong theory," Phys. Rev. D 57, 5032, 1998; hep-th/9902039.

[9] A. O. Barut and D. Villarroel, "Radiation reaction and mass renormalization in scalar and tensor fields and linearized gravitation," J. Phys. A 8, 156, 1975.

[10] P. Havas and J. N. Goldberg, "Lorentz-invariant equations of motion of point masses in the general theory of relativity," Phys. Rev. 128, 398, 1962.

[11] E. G. P. Rowe and G. T. Rowe, "The classical equation of motion for a spinning point particle with charge and magnetic moment," Phys. Rep. 149, 287, 1987. 